\title{
Crystal lattice engineering the novel substrates for III-nitride-oxide heterostructures
}

\author{
V. Osinsky ${ }^{1}$, O. Dyachenko ${ }^{2}$ \\ ${ }^{1}$ Institute of Microdevices, Kyiv, Ukraine; e-mail: osinsky@voliacable.com. \\ ${ }^{2}$ Kyiv Polytechnic Institute, Kyiv, Ukraine
}

\begin{abstract}
In this work, we firstly investigated controlling the lattice parameter of IIIoxides used as substrates for III-nitrides heterostructures. It was shown that the atomic content change in III-sublattice gives large possibilities for precise cation controlling the lattice parameters. The developed technique is promising to make ideal substrates in IIInitride epitaxy of LED, LD and transistors with a high quantum efficiency and small noise. This technology can be realized using MBE, MOSVD or CVD chloride-hydride epitaxy with computer driving.
\end{abstract}

Keywords: III-nitrides, III-oxides, cation operation, bandgap, matrix.

Manuscript received 04.03.10; accepted for publication 25.03.10; published online 30.04.10.

\section{Introduction}

The most urgent problem in technology of III-nitride heterostructures is the high density of defects and strains caused by mismatch of the lattice parameter and thermal expansion coefficient between III-nitride nanolayers and most commercially available substrates from sapphire, which is electrically insulating and stops current spreading. It has a low thermal conductivity, which limits heat dissipation.

$\mathrm{SiC}$ substrates are better but have too high cost. Homoepitaxial structures on bulk GaN or AlN substrates have substantially less defects, but large-area low-cost $\mathrm{GaN}$ wafers is not available nowadays.

The low-cost and large-area $\mathrm{Si}$ substrates have a great advantage of monolithic integration with $\mathrm{Si}$ integrated circuits [1], but light extraction and quantum efficiency of LED structures are less.

We investigated novel substrates for III-nitrides heterostructures on compound III-oxides like (AlGaIn $)_{2} \mathrm{O}_{3}$ and their solid solutions (SS). Their advantages are as follows: ideal lattice parameter matching, high electrical conductivity, optical transparency in all the visible and UV ranges, low cost and technological harmonization with III-nitrides.

Precise driving the energy gap and lattice parameters in LED heterostructures was realized by changing their main composition in quaternary system InGaAsP [2], and it was spread for different multicomponent systems $\mathrm{A}^{3} \mathrm{~B}^{5}$, including III-nitrides [3].
Nevertheless, all the commercially III-nitride LEDs are predominantly made now on (0001) sapphire plane or $\mathrm{SiC}$ substrates. This direction is polar, which creates strong polarization-induced internal electric field leading to a reduced overlap between the electron and hole wavefunctions as well as low radiative recombination time. In order to overcome this problem, the growth of the III-nitride structure along nonpolar directions is preferable. But the growth along nonpolar directions offers a number of advantages over devices currently grown along the (0001) direction. In this sense, it is interesting to grow III-nitride heterostructure on III-oxide substrate, for example, $\alpha$-plane $\mathrm{GaN}$ can be grown using an $\alpha$-plane $(\mathrm{AlGa})_{2} \mathrm{O}_{3}$ substrate instead of $\alpha$-plane of sapphire.

\section{Lattice parameter cation operation}

$\mathrm{Al}, \mathrm{Ga}$, In cations, like to those in III-V compound semiconductors, are tetrahedrally coordinated with the oxygen anion via $\mathrm{sp}^{3}$ covalent bonding; however, the IIInitride bond is substantially more ionic in its nature. Also it seems interesting to grow $\alpha$-plane of GaAlInN on r-plane of sapphire. In addition to differences in material quality that arise from differences in lattice matching and growth conditions, the orientation of the quartzite crystal structure on the III-oxide substrate also influences the LED and laser properties of the fundamental ways by changing the orientation of the light extraction and resonator formation with respect to the plane of the wafer. 


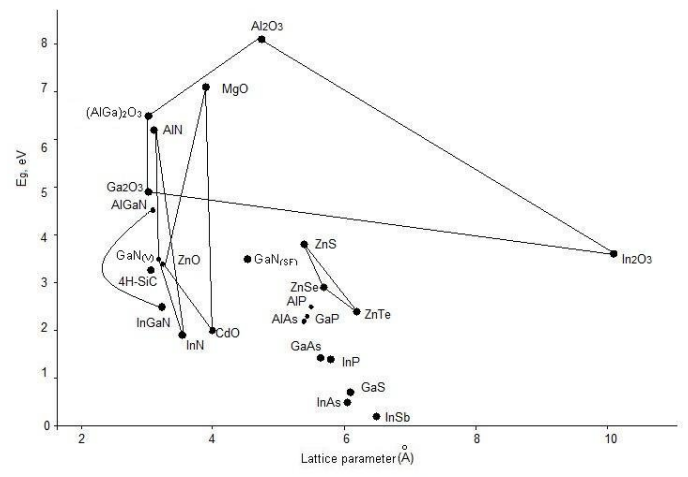

Fig. 1. Bandgap versus lattice parameter diagram showing the relationship between III-oxides and III-nitrides.

Bandgap and lattice structure engineering as well as the possibility to form heterostructures is crucial for carrier and optoelectronic limitations in LEDs and lasers. $\mathrm{Al}$ increases the bandgap in $\mathrm{Ga}_{2} \mathrm{O}_{3}$ matrix, while In decreases the bandgap. The solid solubility limit of In in $\mathrm{GaN}$ is about $12 \%$. However, using nonequilibrium deposition techniques like ionic or molecular-beam epitaxy, $\mathrm{Al}_{\mathrm{x}} \mathrm{Ga}_{1-\mathrm{x}-\mathrm{y}} \mathrm{In}_{\mathrm{y}} \mathrm{N}$ with $\mathrm{x}, \mathrm{y}=0 \ldots 1$ can be grown while maintaining the quartzite crystal structure. Usually, nonequilibrium growth gives phase separation and formation of crystallites. The interesting aspect of the phase separation of multicomponent alloys is that the bandgap of binary or ternary crystallites is different. Thus, the radiation and absorption have as broad band as white light does. Then the bandgap of surrounding

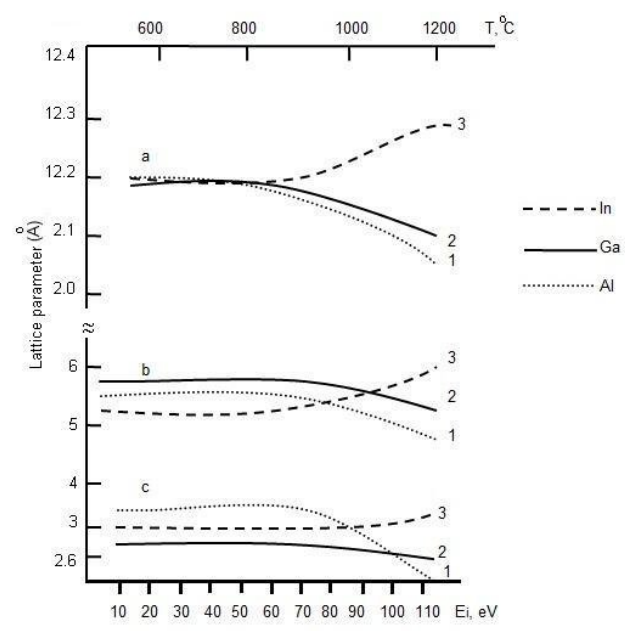

Fig. 2. Ion energy and heat-treatment dependence of the lattice parameter for: $1-\left(\mathrm{Ga}_{\mathrm{x}} \mathrm{Al}_{1-\mathrm{x}}\right)_{2} \mathrm{O}_{3} \mathrm{SS}, \mathrm{Al}^{3+}$ incorporated into $\beta-\mathrm{Ga}_{2} \mathrm{O}_{3}$ matrix; $2-\left(\mathrm{Al}_{\mathrm{x}} \mathrm{Ga}_{1-\mathrm{x}}\right)_{2} \mathrm{O}_{3} \mathrm{SS}, \mathrm{Ga}^{3+}$ incorporated into $\mathrm{Al}_{2} \mathrm{O}_{3}$ (nano-sapphire) matrix; $3-\left(\mathrm{Ga}_{\mathrm{x}} \mathrm{In}_{1-\mathrm{x}}\right)_{2} \mathrm{O}_{3} \mathrm{SS}, \mathrm{In}^{3+}$ incorporated into $\mathrm{Ga}_{2} \mathrm{O}_{3}$ matrix; material matrix is large, and quantum dots form. Examining the bandgap versus lattice parameter diagram, one finds that this allows one to create alloys with bangaps that range from 1.3 to $6.3 \mathrm{eV}$, as shown in Fig. 1. In this paper, the cation precise driving the lattice parameters of III-oxides was proposed. Binary III-oxides are $\mathrm{Al}_{2} \mathrm{O}_{3}, \mathrm{Ga}_{2} \mathrm{O}_{3}$ and $\mathrm{In}_{2} \mathrm{O}_{3}$. All of them are widely used in optoelectronic and microwave devices with their native crystal structures and lattice parameters [5,6]. Atomic changing in III-sublattice gives more complicated structural and physical-and-chemical characteristics in comparison with one type of atoms due to different atomic sizes and mass, which changes chemical bonding in crystal lattice. X-ray analysis of atomic dislocation in $(\mathrm{AlGa})_{2} \mathrm{O}_{3}$ gives large dislocation change of more light atoms of $\mathrm{Al}$ in comparison with more heavy and larger atoms of gallium. Dislocation change of compound oxides has an intermediate position.

The optical energy gap investigation shows a dependence of $E_{g}$ on the atomic-ion energy (temperature) of solid solution during its preparation. At a low atomic energy (temperature less $750{ }^{\circ} \mathrm{C}$ ), the lattice parameter of $\beta-(\mathrm{AlGa})_{2} \mathrm{O}_{3}$ stays constant. But when the ion energy of $\mathrm{Al}^{3+}$ is increased up to $E_{i}(\mathrm{Al})=$ $40-50 \mathrm{eV}\left(T>850{ }^{\circ} \mathrm{C}\right)$, the lattice parameter with the ion of $\mathrm{In}^{3+}$ is decreased in $a, b$ and $c$ axis. This can be explained by incorporated diffusion of $\mathrm{Al}^{3+}$ and $\mathrm{In}^{3+}$ in $\mathrm{Ga}_{2} \mathrm{O}_{3}$ matrix, which has been observed at ion energies 50 to $60 \mathrm{eV}$ and temperatures above $1100{ }^{\circ} \mathrm{C}$ [7]. At higher energies of $\mathrm{Al}^{3+}$ ions, there is a decrease in the SS lattice parameters, while an increase of the lattice parameter is observed when the large ion $\mathrm{In}^{3+}$ is incorporated with a high ion energy (or temperature). Since the ionic radius of $\mathrm{Al}^{3+}(0.54 \AA)$ is smaller than that of $\mathrm{Ga}^{3+}(0.62 \AA)$, the lattice parameter would be reduced, if $\mathrm{Al}$ substitutes for $\mathrm{Ga}$ site in $\beta-\mathrm{Ga}_{2} \mathrm{O}_{3}$ lattice. If $\mathrm{Al}^{3+}$ and $\mathrm{In}^{3+}$ are incorporated into SS lattice, $E_{g}$ would increase with $\mathrm{Al}^{3+}$ content and decrease with $\mathrm{In}^{3+}$ content. This gives a possibility to precisely regulate lattice parameters of SS (Fig. 2).

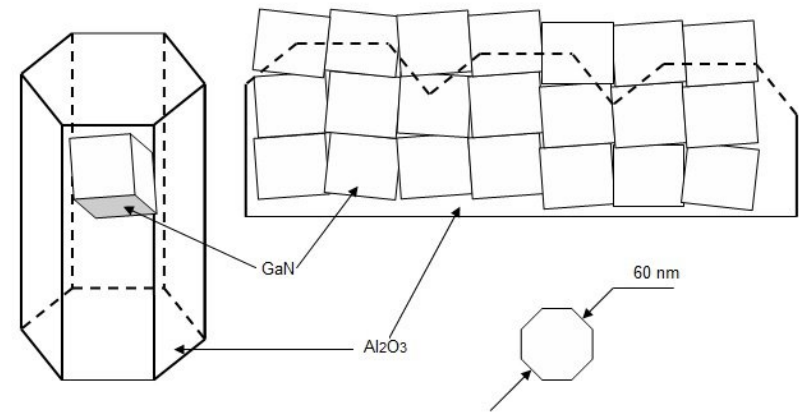

Fig. 3. The cubic GaN nanostructure growth on the internal surfaces of nano-sapphire pores. 


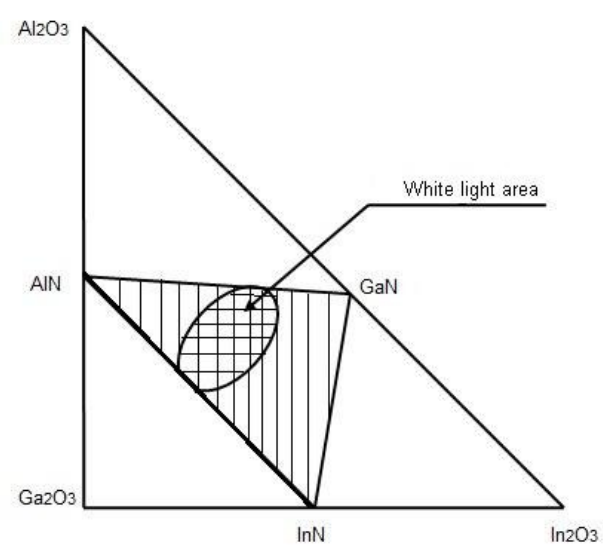

Fig. 4. Lattice parameter diagram for III-nitride-oxide heterostructures.

It has previously been shown [8] that selective hydride vapor phase epitaxy (HVPE) of $\mathrm{GaN}$ on $\mathrm{Al}_{2} \mathrm{O}_{3}$ nanostructures (nano-sapphire) gives nonpolar cubic GaN with a small density of defects. A new model of this growth can be proposed, if we take into account the crystal nanostructure (Fig. 3) inside nanoholes of $\mathrm{Al}_{2} \mathrm{O}_{3}$ film. There is a lattice parameter equal to $4.75 \mathrm{~nm}$ in nano-sapphire, which stimulates preferential crystallization of cubic GaN with the same crystal parameters $(a=b=c=0.45 \mathrm{~nm})$. The lattice parameter engineering proposed in this paper gives a good possibility to drive the nanostructure of III-nitridesoxides (Fig. 4) for high quality LED, LD and transistors.

\section{Conclusion}

In conclusion, the precise driving the lattice parameters of III-nitrides-oxides is possible to obtain high quality multilayer heterostructures for optoelectronic and electronic devices. III-oxides can be used as ideal substrates for epitaxy of III-nitrides with the same lattice parameter, which can give defectless structures. For LED and LD structures, it means that the internal quantum efficiency can reach $100 \%$. FETs and HEMT will have very small noise, as well as high-power and high-frequency transistors would have maximized parameters. This technology can be realized by MBE, MOSVD or CVD chloride-hydride epitaxy with computer driving.

\section{References}

1. V. Osinsky, D. Murchenko, H. Honarmand, $\mathrm{Si} / \mathrm{A}^{3} \mathrm{~B}^{5}$ one chip white LED sources integration // Semiconductor Physics, Quantum Electronics and Optoelectronics, 12 (3), p. 240-250 (2009).

2. N. Sirota, V. Osinsky, Radiation of $p-n$ junction based on solid solution of indium phosphategallium arsenide crystals // Trudy Academii Nauk SSSR, ser. fizich. 171-172, p. $317-319$ (1966), in Russian.

3. V. Osinsky, V. Verbitsky, Band gap engineering: ion realization of virtual quantum heterostructure of nanoelectronics // Optoelectronic informationpower technologies №1, p. 169-183 (2001).

4. N.N. Sirota, Some problems of chemical bonding in semiconductor, in: Chemical Bonding in Semiconductor and Solid Systems. Nauka i tekhnika, Minsk, 1965, p. 12-45 (in Russian).

5. O. Dyachenko, V. Osinsky, The operation of properties of complicated III-oxides nanocrystal elements in devices based on $\mathrm{Ga}$, In, $\mathrm{Al}$ nitrides. To be published in Electronics and communications.

6. J. Muth, V. Osinsky, Optical Properties of $\mathrm{ZnO}$ Alloys. Wide Bandgap Light Emitting Materials and Devices. Edited by G.F. Neumark, I.L. Kuskovsky and H. Jiang. Wiley-VCK, 2001, p. 179-204.

7. G.A. Battiston, R. Gerbasi, M. Potchia et al. // Thin Solid Films 279, p. 115 (1996).

8. A. Poliakov, V. Osinsky, G. Gorokh et al., Nonpolar GaN grown on Si by hydride vapor phase epitaxy using anodized Al nanomask // Appl. Phys. Lett. 94, 022114-1 - 022114-3 (2009). 